\title{
Accretion Disks of Bounce-Back CVs
}

\section{Gagik Tovmassian and Sergey Zharikov}

Instituto de Astronomia, UNAM, Ensenada, BC, Mexico

email: gag, zhar@astrosen. unam.mx

\begin{abstract}
We explore conditions and structure of accretion disks in the short-period Cataclysmic Variables, which have evolved beyond the period minimum. We show that the accretion disk in a system with extreme mass ratio grows in the size reaching 2:1 resonance radius and are relatively cool. They also become largely optically thin in the continuum, contributing to the total flux less than the stellar components of the system. In contrast, the viscosity and the temperature in spiral arms formed at the outer edge of the disk are higher and their contribution in continuum plays an increasingly important role. We model such disks and generate light curves which successfully simulate the observed double-humped light curves in the quiescence.
\end{abstract}

Keywords. Cataclysmic variables, accretion disk, period minimum.

\section{Introduction}

The bounce-back systems (BBs) are Cataclysmic Variables (CVs) evolved beyond the minimum period limit, which is reached when the secondary star becomes of a substellar mass and partially degenerate. The secondary's thermal timescale, while it approaches the period minimum, increases faster than the timescale on which it loses mass. The star is driven out from the thermal equilibrium and at some point its radius stops contracting in response to mass loss and even grows. The size of the binary orbit increases in order to accommodate the secondary, so there is a change in the direction of the system's period evolution (Paczynski \& Sienkiewicz 1981). The age of the Galaxy is old enough for significant fraction of the current short-period CV population to have evolved past the orbital period minimum (Kolb \& Baraffe 1999).

Fig. 1 illustrates the current concept of evolution of CVs and displays position of bounce back systems on the mass-transfer rate and mass-ratio to the orbital period diagrams. The size of accretion disk (AD) is a function of mass ratio and the resonance radii are marked on the left panel. The systems below the 3:1 threshold show super-humps during super-outbursts as a result of precession of a tidally distorted disk (Papaloizou \& Pringle 1979) and are identified as SU Uma stars. Characteristics of CVs change as they approach the period minimum. Instead of frequent outbursts followed by cyclical super-outburst, the systems undergo only very powerful super-outburst with a time gap between them reaching three decades as in the case of WZ Sge, the prototype of similar systems. WZ Sge during super-outburst has shown a double humped light curve (Patterson et al. 2002). Such behavior, i.e. two humps per orbital period in the light curve was predicted by Lin \& Papaloizou (1979), as a result of formation of spiral density waves in the outskirts of the disk under the influence of $2: 1$ resonance. So, it is expected that the systems below the 2:1 threshold should form spiral arms also in the quiescence. Most systems enclosed in the box Fig. 1 show permanent double humped light curve in quiescence of varying amplitude. They also have a mildly blue continuum with relatively weak Balmer emission lines from the AD surrounded by broad absorptions formed in the atmosphere of WD. Four objects (V455 And, AL Com, SDSS 0804 and EG Cnc) produced WZ Sgetype super-outbursts in the near past. Estimates of system parameters show a presence 

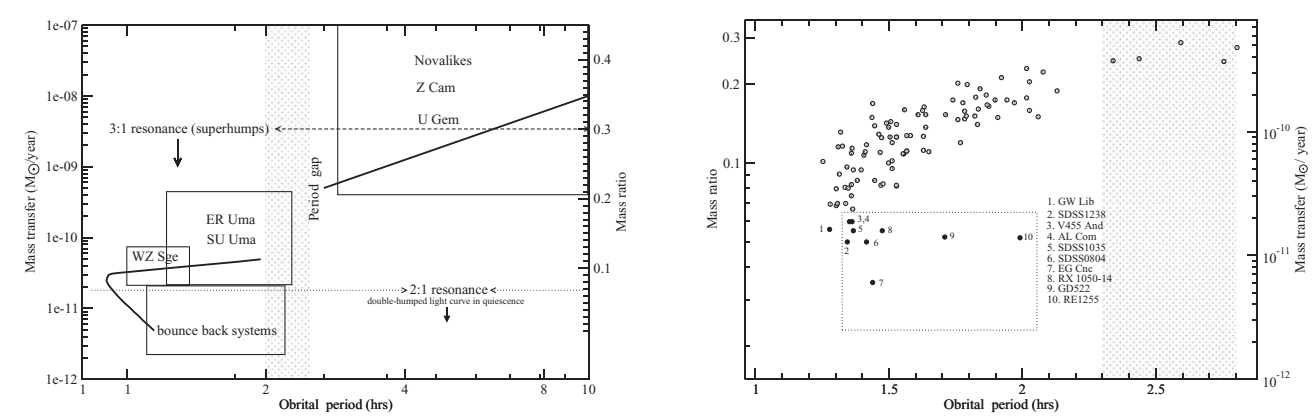

Figure 1. Plot of the mass ratio and the mass transfer rate vs the orbital period. Left) Schematic distribution of CV types: (Right) The BB candidates are enclosed in the box.

of relatively cool $\left(\mathrm{T}_{\text {eff }} \approx 12000 \pm 1000 \mathrm{~K}\right)$, massive white dwarf $\left(\mathrm{M}_{1} \sim 0.9 \mathrm{M}_{\odot}\right)$ and an extremely low value of mass ratio $q \leqslant 0.06$, which assumes a brown dwarf as a secondary. Some new phenomena were discovered in bounce-back candidates not seen anywhere else in CVs. One is the long 3.5h spectroscopic period not related to the orbital in V455 And (Araujo-Betancor et al. 2005), second, there are the "brightenings" permanently observed in SDSS1238 and occasionally seen in SDSS0804 before it went through a super-outburst in March, 2006 (Zharikov et al. 2006). Finally, there are "mini-outbursts" observed in SDSS0804 (Zharikov et al. 2008) about one year after the super-outburst of 2006.

\section{Nature of the accretion disk in bounce-back systems}

We analyze the AD structure of BB candidates based on spectral and IR photometric observations of SDSS1238 and SDSS0804 in the quiescence. The conditions of the disk are inferred from the Doppler tomography (Fig. 2) displaying the internal structure, and, the estimate of AD contribution in the total flux of the systems from the spectral energy distribution (SED; see Fig. 3). For both objects the Doppler tomograms show two prolonged structures, one overlapping with the bright spot, another of similar size at the opposite side of the disk. Similar structures were detected in the quiescence spectra of the BB candidate SDSS 1035 (Southworth et al. 2006) and WZ Sge during super-outburst (Baba et al. 2002). Smooth particle hydrodynamics models of AD developed by Kunze \& Speith (2005) demonstrate formation of spiral density waves in a very illustrative form. We used their code to produce models of ADs with parameters corresponding to the BBs. We produced Doppler tomogram from the model disk. The results along with the observed tomograms of two BBs are shown in Figure 2.

Synthetic Doppler map satisfactory reproduces the observed ones if density is increased within the spiral arms. Most particles in the $\mathrm{AD}$ are on periodic orbits, which are the most favorable from the point-of-view of viscosity. The BBs as well as WZ Sge stars are believed to have low viscosity disks. However, the resonance dispatches some particles onto aperiodic orbits creating viscosity perturbations, which will create density/heat excess. We have to assume that the optical depth reaches critical unity in these density waves making them prominent in the BB systems as a result of contrast, since the rest of the $\mathrm{AD}$ is probably largely optically thin and has small contribution to the continuum.

The visibility of both stellar components is good evidence of insignificant contribution of the AD to the total optical-IR flux of the system. The optical-infrared SED of SDSS 1238 \& SDSS 0804 fitted by the three component model (WD, a brown dwarf BD, and a standard $\mathrm{AD}$ ) are shown in Figure 3 . The model presumes very small contribution from the disk ( $50 \%$ or less) to the optical flux of the system compared to a ordinary CVs 

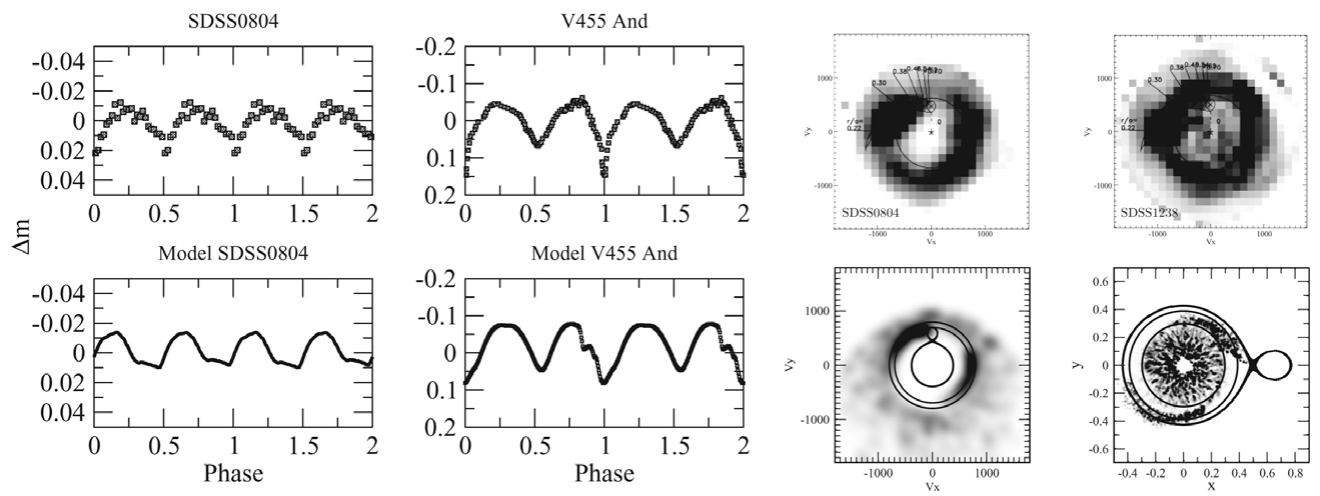

Figure 2. Left) Sample light curves of BBs and models reproducing them. (Right) The Doppler tomograms of SDSS0804 and SDSS1238 (top) and their simulation (bottom).

in which the AD dominates. The main uncertainty of the model is the slope of the AD continuum. Zharikov et al., 2012 used a flatter AD spectrum to fit the observed SED of SDSS 0804, but that does not change basic conclusion on the system composition and the requirement that the $\mathrm{AD}$ is largely transparent in the continuum.

It is known that ADs in CVs develop optically thin outer regions for mass transfer rates below about $10^{-8} \mathrm{M}_{\odot} \mathrm{yr}^{-1}$ (Williams 1980). Tylenda (1981) suggests that an increase in the radius of the $\alpha$-model disk always increases the role of the thin region. For $\alpha \leqslant 1$ the thin part of the disk is rather cool $(\leqslant 6000 \mathrm{~K})$. As a large part of the AD is optical thin in continuum, the disk temperature can drop below 5000K (Dumont et al. (1991). Cannizzo $\&$ Wheeler (1984) studied the vertical structure of a steady-state, $\alpha$-model thin AD for an accreting object of $1 \mathrm{M}_{\odot}$. They found that for low accretion rates, the disk structure is optically thin. For $0.01<\alpha<1$, the solution of disk equations can be double-valued with high $(\sim 5000 \mathrm{~K})$ and low $(\sim 2000 \mathrm{~K})$ temperature branches. For $\alpha>0.1$ a warm solution is possible in the inner region of the $\mathrm{AD}$, but the disk annuli at larger radii will be in a cold state with $T<2000 K$. Only the low temperature solution exists for $\alpha \approx 0.1$. As $\alpha$ decreases with temperature, a tendency to develop cold solutions in quiescence is enhanced. Until now, models of disk emission spectra for such cool disks have not been calculated, the nearest approach is a flat spectrum in the range $3000-10000 \AA$ for the model of the cool ( $\mathrm{T}=5000 \mathrm{~K}, \alpha=0.03) \mathrm{AD}$ (Idan et al. 2008).

But, even in such cool and largely transparent disk the inner parts will be bright in the continuum and their contribution would be enough to cancel variability introduced by spiral waves.

In the case of WZ-Sge, various authors support the idea that the inner part of the disk need to be void in order to explain the long recurrent time for super-outbursts
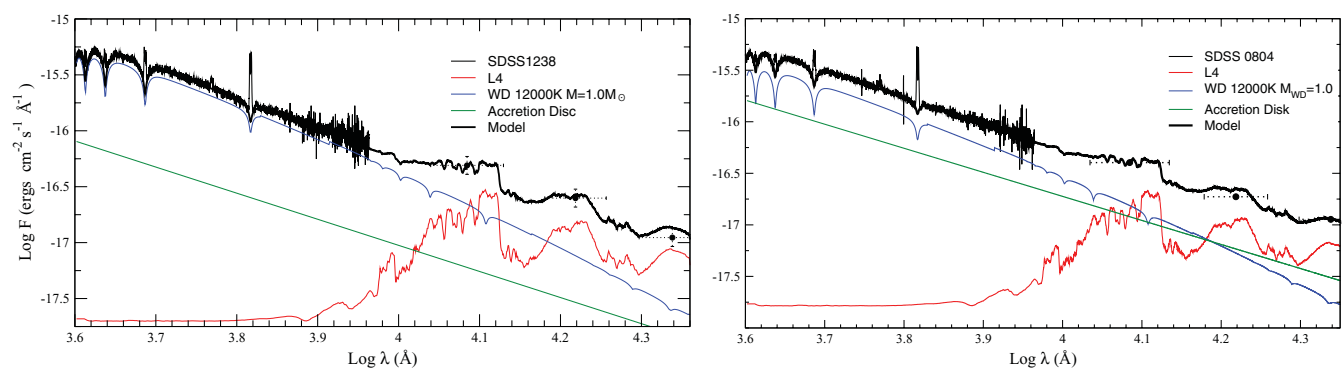

Figure 3. The SED of SDSS1238 (left) \& SDSS 0804 (right) and results of model fits. 
(see Kuulkers et al.(2011) and reference therein). It should be noted that even a weak magnetic field ( $\leqslant 1$ MGauss) is enough to form a cavity in the inner part of the AD in BBs with very low accretion rates. Such weak fields might be difficult to detect. Among bounce back candidates V455 And is known to be an intermediate polar.

Actually, V455 And is a very interesting case: the radial velocities of its spectral lines are modulated with two different periods. One is the usual S-wave arising from a bright spot at the outer edge of the disk and seen in the center of the line. The other is a long 3.5 hour period appearing in the wings of the lines and obviously originating in the inner part of the disk (Araujo-Betancor et al. 2005). But neither spot has much influence in the light curve (see Fig. 2). Usually, high inclination CVs, show pronounced hump prior to the eclipse, which arises in the bright spot of impact of the mass transfer stream with the disk. In the light curve of V455 And two smooth humps appear with partial eclipse, but no characteristic pre-eclipse hump. This may only signify, that the spot is emitting in emission lines, but not much in the continuum. As for the inner spot, the only other system that shows the same kind of long spectroscopic period is FS Aur. But FS Aur in a stark difference to V455 And exhibits a wild photometric variability and one of the significant periodic signal in the photometry is directly related to the long spectroscopic period. FS Aur however has very luminous AD which totally over shines stellar components.

We developed a geometric model of CV disks having two spiral density waves in outer annuli of a thin AD to model light curves of BBs (Zharikov et al. 2012). The model apart from the standard components (WD, secondary star, accretion stream and bright spot) takes into account positions of the bright structures in the Doppler maps, according to the description of the spiral density waves in Hachisu et al. (2004). From our models, we find that the shape and the amplitude of the double hump light curve can be realistically re-produced as can be seen from Fig. 2, depending on the parameters chosen for the two spiral density waves, the disk temperature distribution, the size of the inner cavity, and the outer radius of the disk. We also find that the amplitude of the double-humped variability decreases with decreasing temperature gradient in the spiral density waves and/or the decline of the system's inclination.

\section{References}

Araujo-Betancor, S, et al. 2005, A\&GA, 430, 629

Baba, H., et al. 2002, PASJ, 54, L7

Cannizzo, J. K. \& Wheeler, J. C. 1984, ApJS, 55, 367

Dumont, A. M., et al. 1991, A\&SA, 242, 503

Gänsicke, B. T., et al. 2009, MNRAS, 397, 2170

Hachisu, et al. 2004, ApJL, 606, L139

Idan, I., et al. 2008, New A. Rev., 51, 759

Kolb, U. \& Baraffe, I. 1999, MNRAS, 309, 1034

Kuulkers, E., et al. 2011, A\&SA, 528, A152

Lin, D. N. C. \& Papaloizou, J. 1979, MNRAS, 186, 799

Paczynski, B. \& Sienkiewicz, R. 1981, ApJL, 248, L27

Patterson, J., Masi, G., Richmond, M. W., et al. 2002, PASP, 114, 721

Papaloizou, J. \& Pringle, J. E. 1979, MNRAS, 189, 293

Southworth, J., et al. 2006, MNRAS, 373, 687

Tylenda, R. 1981, Acta. Astron, 31, 127

Williams, R. E. 1980, ApJ, 235, 939

Zharikov, S. V., et al. 2006, A\&SA, 449, 645

Zharikov, S. V., et al. 2008, A\&SA, 486, 505

Zharikov, S. V., et al. 2012, submitted to $A \& A$ 